\title{
Monatsschrift fur
}

Psychiatrie imd

Neurologie

Begriindet von C. Wernicke und Th. Ziehen

Fortgefuhrt von K. Bonhceffer

\section{Journal Mensuel de Psychiatrie} et de Neurologie - Monthly

Journal

\section{of Psychiatry and Neurology}

Collaborators:

B. Brouwer

Amsterdam

L. Benedek

Budapest

0. Diethelm

New York

E. Kahn New Haven

H. W. Maier

Zurich

G. Pamboukis

Athen

H. Steck 
Lausanne

C. U. Ariens Kappers

Amsterdam

E.

Carp

Leiden

B* Jacobowsky

Upsala

J. Lhermitte

Paris

F. Morel

Geneve

J. Staehelin

Basel

L. van Bogaert

Anvers

H. Helweg

Kopenhagen

J. Lupine

Lyon

A. Meyer

Baltimore

H. Schou

Dianalund

R. Gjessing

Oslo

G. Langfeldt

Oslo

E. Map other

London

C. Pfersdorff

Strasbourg

C. I. Urechia G, Vermeylen

Cluj

Bruxelles

Editor: J. KLAESI, Bern 


\section{Vol. IOS}

\section{$\wedge^{I} \mathrm{~T} \%$ \\ -If K.}

d 9)

\section{BASEL (Schweiz)}

Holbeinstrafie 22

S. K A R G E R

NEW YORK

215 Fourth Arenae

Alle Rechte vorbehalten

Printed in Switzerland Buchdruckerei Winterthur A.-G.

\section{INDEX:}

Angyal, L. v. and K. Gydrfas, Contributions to the Vaccine Therapy of Radicular Symptoms in Tabes

Bogaert, L. van, M. Maere et E. de Smedt, Sur les formes familiales precoces de la maladie d'Alzheimer. 
Brouwer, B., Untersuchung des Gehirns nach einer scheinbar volligen operativen Entfernung eines Glioms.

Buchanan, A. R., Vestibular Harmony and Disharmony in Guinea Pigs with

Lesions in the Cerebellum and Brain Stem

Bumbacescu, vide Urechia, C. I.

Danjou, $P$., vide Fame, $A$.

Favre, A. et P. Danjou, Delire sensitif psychogene, echec par l'insulinothera-

pie, guerison par une cure de sommeil

Griinthal E. und K. Hartmann, Beitrage zur Kenntnis des extrapyramidal-motorischen Systems. I. Uber einen Fall von Hemichorea mit Stria-tumherd

- - und R. Stahli, Beitrage zur Kenntnis des extrapyramidal-motorischen

Systems. II. Uber Torsionsdystonie nach Encephalitis epidemica . 115

— und 0. Wenger, Erganzendc Untersuchungen und Bemerkungen zuder Arbeit: Nachweis von Erblichkeit bei der Alzheimerschen Krankheitusw. in Band 101 dieser Monatsschrift

Gydrfas, K., vide Angyal, L. v.

Hartmann, K., vide Griinthal, E.

Heymann, K., Psychose und Farbenblindheit

Hoff, H. and J. A. Shahy, Two Cases of Menstrual Psychoses Aetiology and

Therapy......

Hordnyi-Hechst, B., Uber die anatomisehe Lokalisation katatoner Erschei-

nungen

Kafka, V., Zum Problem der Liquorkategorien

Maere, M., vide Bogaert, L. van.

Manta, I., vide Urechia, C. I.

Montmollin, R. de, vide Morel, F.

Morel, F. et R. de Montmollin, Psychose Ethylico-Pellagreuse...

Miiller, M., vide Urechia, C. I.

Retezeanu, Ah, vide Urechia, C. I.

Rosenthal, C, Uber das Auflreten von halluzinatorisch-kataplektischem Angst-

syndrom, Wachanfalien und ahnlichen Storungen bei Schizophrenen 11

Scheinker, I., Zur Frage Gehirntumor und Schadeltrauma

— Zur Histopathogenese der Hirnapoplexie bei Hypertonic

— Encephalitis optica unter dem Bilde eines Tumor cerebri $\quad$... 353

Schoch, M. A., Uber Zusammenhange zwischen tertiarer Lues, Arznei-Into-

leranzen, Neurorezidiven und progressiver Paralyse.

Schou, H. J., Hauttemperatur bei manisch-depressiven Fsychosen .... 327

Shaby, J. A., vide Hoff, $H$.

Smedt, E. de., vide Bogaert, $L$.

Stahli, $R$., vide Griinthal, E.

Urechia, C. I., I. Manta et Bumbacescu, Quelques recherches (experimentales)

biologiques et histologiques chez les brules

- Al. Retegeanu et M. MUUer, Les complications nerveuses du charbon(encephalo-meningo-myelite) 336-

Wenger, 0., vide Griinthal, E.

Wyrsch, J., Tiber die Psychopathologie einfacher Schizophrenien. 
Margarete v. Andics, Uber Sinn und Sinnlosigkeit des Lebens. Auf Grand

von Gesprachen mit geretteten SelbstmSrdern ............................................................... 127

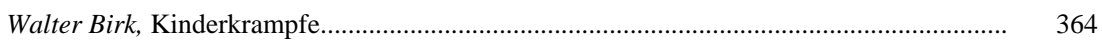

M. Dorer, Charakter und Krankheit. Ein Beitrag zur Psychologie der

Enzephalitis epidemica.

J5. Dukor, Das schweizerische Eheverbot fur Urteilsunfahige und Geistes-

kranke.

M. H. Goring, Uber seebsch bedingte echte Organerkrankungen . . . 191

C. G. Jung, Wandlungen und Symbole der Libido $\quad$ …........................................................ 190

Knud H. Krabo\% Studies on the Morphogenesis of the Brain in Reptiles126Gabriel Langfeldt, The Schizophreniform States. A katamnestic study,

based on individual re-examinations . MB.....

K. Leonhard, InvollBye und idiopathische Angstdepression in Klinik und Erbhchkeit

G. H. Monrad-Krohn, The Clinical EgKamination of the Nervous System 64

Varia .W\&.....

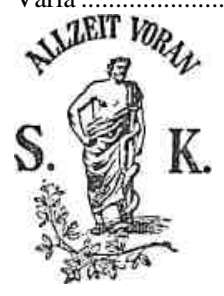

\section{$1890 ;-$ V. S. KARGER}

40

VERLAG FOR MEDIZIN UND NATURWISSENSCHAFTEN

A

m 1. April 1940 sind 50 Jahre seit der Griindung des medizinischen . Verlages $\mathrm{S}$. Karger vergangen.

Herr S. Karger hatte zunachst die Ahsicht, fur Arzte und Studierende kurze, von anerkannten Forschern verfafite Anleitungen herauszugeben, um durch sie die fur die Praxis notwendigen wissenschaftlichen Grund-lagen und Erkennrnisse dem Lernenden auf moglichst hohem Niveau zu vermitteln. Nach dem vollen Erfolg dieser Biicher wurde schon von 1894 an auch eine Reihe von Zeitschriften geschaffen, die eine Stellung zwischen den rein wissenschaftlichen Archiven und den vorwiegend fiir die Praxis hestimmten Blattern einnehmen sollten. Diese Zeitschriften fuhrten sich bald auf der ganzen Welt ein und nahmen an Zahl zu, so dafi die person-hche Initiative von Herm S. Karger auch hier einen vollen Erfolg feiem durfte. 
Seit seinem Tode im Jahr 1935 Avird der Verlag im Sinne des Griin-ders fortgefuhrt.

Im Jahre 1937 erfolgte die Ubersiedelung der Firma nach Basel. Hier wurde mit HiMe der unterzeichneten Herausgeber die Verwirklichung der Idee weiterverfolgjMin den Zeitschriften eine Art Zentralstelle fiir eine Internationale Zusammenaibeit auf den medizinischen Spezialgebieten zu schaffen. Die Zeitschriften sollten einem fortlaufenden Gedankenaustausch dienen, etwa in der Art, wie er durch die internationalen Kongresse er-moghcbt wird.

Es ist die Uberzeugung des Verlages und der Herausgeber, dafi eine solche Zusammenarbeit auf breitester internationaler Basis heute wichtiger ist als je. Deshalb wurde nicht nur den alten Zeitschriften eine neue Form gegeben, sondern es wurden auch neue derartige internationale Organe gegriindet. Sie hahen sich trotz der ungiinstigen aufieren Umstande schon in kurzer Zeit sehr gut eingefuhrt; es diirften zahlrefraie personliche Zustimmungen, die von Forschern der ganzen Welt einlaufen, wohl ein Zeichen dafiir sein, dafi wir uns hier auf dem richtigen Wege befinden.

Den unterzeichneten Herausgebern ist es ein Bediirfnis, dem Verlag S. Karger anlafilich seines 50jahrigen Bestehens fur das trotz der schweren Zeiten stets verstandnisvolle Eingehen auf ihre Wiinsche zu danken und der Hoffnung Ausdruck zu geben, es moge den Bestrebungen des Verlags audi weiterhin ein voller Erfolg beschieden sein.

n the 1st of April 1940, the Medical Publishing House, S. Karger will celebrate its 50th anniversary. The Founder, Mr. S. Karger originally intended to publish short textbooks for medical practitioners and students written by eminent scientists, in order to furnish the learner with the scientific fundamentals and knowledge necessary for practice. After these books had attained a full measure of success, a series of scientific journals were founded in 1894 intended to have an intermediate position between the purely scientific archives and the journals devoted to the practice of medicine. These soon spread throughout the whole world and increased in number, so that the personal initiative of Mr. S. Karger was rewarded with success.

Since his death in 1935, die Publishing House has been continued on the principles of its Founder.

In the year 1937, die Company moved to Basle. With the assistance of the undersigned Editors the idea was carried still furtiher forward to make the journals a sort of central organ for international cooperation in the domain of medical specialities. The journals are meant to serve as a channel for the conffiuous exchange of ideas in a manner similar to that made possible by thelttiternational Congresses.

The Publishers and Editors are convinced diat such cooperation on a wide international basis is more than ever essential today. Therefore, not only were die old journals given a new form, but new international organs have been 
called into being as well. In spite of die unfavourable circumstances obtaining, these periodicals made their way in a short space of time and die many personal acknowledgements received from scientists all over the world may well be proof diat we are on die right road.

The undersigned Editors feel themselves constrained to offer the Publishing House S. Karger, on the occasion of die 50th anniversary of die foundation thereof, dieir sincerest thanks for die sympadietic favour ever accorded to their wishes and to express die hope that in spite of very! difficult times die Firm's endeavours may also in the future meet widi a full measure of success.

a maison d'editions medicates S. Karger fetera le ler avril 1940 le cinquantieme anniversaire de sa fondation.

Apres avoir publie, au debut et avec succes, une serie de precis scien-rifiques destines aux praticiens et qui connurent et coamaissent encore une tres large diffusion, S. Karger lanca, des 1894, plusieurs periodiques medicaux qui ne tarderent pas a se repandre dans tous les pays.

Apres la mort, survenue en 1935, du fondateur de la Maison, et fidele a I'esprit qui 1'avait anime, son successeur fut amene, en 1937, a se fixer a Bale, pour y etablir, avec l'aide de divers redacteurs, un centre de collaboration medicale intemationale qui se revele plus necessaire aujourd'hui que jamais. A la liste des anciens periodiques transformes s'en ajouta de nouveaux; les uns et les autres se rejouissent d'un cercle de collaborateurs et de lecteurs de plus en plus etendu.

Les redacteurs soussignes apportent ici a un editeur sans cesse pre-occupe de „servir" l'hommage de leur felicitations et de leur gratitude.

ANNALES PAEDIATRICI

(Jahrbuch fur Kinderheilkunde)

(Fund. 1857) E. Freudeaberg

DERMATOLOGICA

(TDermatologische Zeitschrift)

(Fund. 1893) W. Lutz

Monatsschrift fiir .

GEBURTSHELFE UND GYNAKOLOGBE

(Fund. 1894) E.Anderes, Th.KoUer

GASTROENTEROLOGIA

(Archiv fiir Verdauungskrankheiten)

(Fund. 1895) A. Hurst, R. Staehelin

Monatsschrift fur

PSYCfflATRIE UND NEUROLOGEE

(Fund. 1897) J. Klaesi

OPHTHALMOLOGICA

(Zeitschrift fur Augenheilkunde)

(Fund. 1899) A. Bruckner, H. Weve

PRACTICA

OTO-RHINO-LARYNGOLOGICA

(Fund. 1907) J. Berberich, E. Schlittler 
RADIOLOGIA CLBNICA

(Radiologische Rundschau)

(Fund. 1932) M. Ludin

CARDIOLOGIA (Fund. 1937) B. Kisch, W. Loffler

BIO-MORPHOSIS (Fund. 1938) H. Bluntschli

CONFINIA NEUROLOGICA (Fund. 1938) E. A. Spiegel

Schweizerische Zeitschrift fur ALLGEMEINE PATHOLOGIE UND

BAKTERIOLOG1E

(Fund. 1938)

A. v. AUjertini

A. Grumbach

H. Mooser 